\title{
Influencia del terroir en el perfil aromático de Tempranillo Blanco en la D.O.Ca. Rioja
}

\author{
Juana Martínez*1, Pilar Rubio-Bretón ${ }^{1}, \mathrm{M}^{\mathrm{a}}$ Eva Vicente $^{1}$ y Enrique García-Escudero ${ }^{1}$ \\ ${ }^{1}$ Instituto de Ciencias de la Vid y del Vino- ICVV (Gobierno de La Rioja, CSIC, Universidad de La Rioja), 26071 \\ Logroño, España
}

\begin{abstract}
Resumen. En los vinos blancos el aroma es uno de los principales atributos de calidad, siendo los compuestos aportados por la uva los que determinan el perfil varietal. Tempranillo Blanco es una variedad derivada de Tempranillo Tinto por mutación natural, que desde el año 2008 está autorizada únicamente en la D.O.Ca. Rioja. Sus vinos manifiestan características organolépticas de alta calidad, con notas afrutadas y florales intensas. En este trabajo se estudió el perfil aromático varietal de Tempranillo Blanco en siete localizaciones geográficas de la D.O.Ca. Rioja durante la campaña 2016. Las parcelas se distribuyeron en las tres subzonas que integran la denominación, con características vitivinícolas diferenciadas debido a las condiciones de clima y suelo. Los resultados obtenidos mostraron diferencias notables en el contenido aromático de la uva procedente de las distintas ubicaciones del viñedo. Los compuestos más abundantes fueron los norisoprenoides (48-70\%), seguidos por los bencenoides (8-29\%) y los aldehídos y cetonas (3-18\%); otros muchos volátiles fueron detectados en menores porcentajes (compuestos $\mathrm{C} 6$, alcoholes, terpenos, ésteres, ácidos...). A pesar de las diferencias observadas, el perfil aromático varietal se mantuvo, por lo que se puede concluir que Tempranillo Blanco es una variedad con amplias posibilidades de adaptación a diferentes entornos vitícolas.
\end{abstract}

\section{Influence of terroir on the aromatic profile of Tempranillo Blanco in the D.O.Ca. Rioja}

\begin{abstract}
Summary. Aroma is one of the main attributes of white wines quality and, in particular, prefermentative aromas provided by the grape are the main responsible for the varietal characteristics. Tempranillo Blanco variety was originated from Tempranillo Tinto by natural mutation, being authorized since 2008 just in the D.O.Ca. Rioja. Its wines show high quality organoleptic characteristics, with intense fruity and floral notes. In this work, the varietal aromatic profile of Tempranillo Blanco was studied in seven geographical locations of the D.O.Ca. Rioja during the 2016 vintage. The plots were distributed in the three sub-areas that make up the Denomination, with distinct vitivinicultural characteristics due to climate and soil conditions. The results obtained showed notable differences in the aromatic content of the grape in the different vineyard plots studied. Norisoprenoids were the most abundant compounds (48-70\%), followed by benzenoids (8-29\%) and aldehydes and ketones (3-18\%), other groups of volatiles were also detected in lower percentage (compounds C6, alcohols, terpenes, esters, acids...). Despite the observed differences, the varietal aromatic profile was maintained stable, so that it could be concluded that Tempranillo Blanco is a variety with wide possibilities of adaptation to different growing conditions.
\end{abstract}

\section{Introducción}

El aroma es uno de los atributos determinantes de la calidad en los vinos blancos, siendo los volátiles aportados por la uva los responsables de su tipicidad [12]. Estos compuestos proceden del metabolismo secundario de las plantas, y su presencia está influenciada por muchos factores, tales como la variedad, las prácticas culturales, el clima, el suelo y la localización geográfica [3-4]. Se encuentran principalmente en el hollejo y la 
pulpa de las bayas, tanto en forma libre (odorantes) como en forma de precursores, ligados a moléculas de glucosa y otros compuestos [5-8]. En la uva se han identificado gran número de compuestos aromáticos pertenecientes a diferentes familias químicas, entre los que destacan monoterpenos, C13 norisoprenoides, bencenoides $\mathrm{y}$ compuestos C6 [9-12]. La familia de los monoterpenos presenta un poder odorífero muy elevado, relacionado con notas florales y afrutadas, son compuestos característicos de las variedades muy aromáticas (Muscat, Riesling, Gewürztraminer...), aunque también se encuentran en menor cantidad en otras consideradas neutras.

El impacto de las condiciones ambientales puede modificar de forma importante la composición volátil de la uva [13]. Numerosos factores (exposición a la luz, temperatura, estado hídrico...) pueden afectar a la evolución de la maduración y a otros procesos que se desarrollan paralelamente, entre los que destaca la síntesis, translocación y acumulación de los aromas varietales [14].

La caracterización aromática de las variedades blancas de difusión internacional ha sido objeto de gran número de estudios [15-17]. Por el contrario, la bibliografía existente sobre las variedades blancas españolas es más escasa [18201, y prácticamente inexistente en algunas de reciente incorporación al viñedo, como es Tempranillo Blanco.

La variedad Tempranillo Blanco se originó a partir de Tempranillo Tinto por mutación natural, siendo detectada en un viñedo de La Rioja en 1988 [21]. Esta variedad se cultiva desde el año 2008 únicamente en la D.O.Ca. Rioja, y en la actualidad es la segunda variedad blanca en cuanto a superficie. Se trata de una variedad de ciclo corto y producción media, que permite elaborar vinos con una composición química equilibrada en grado alcohólico, acidez y contenido polifenólico, por lo que puede ser adecuada para diversificar la elaboración de vinos blancos. Aporta características organolépticas de alta calidad, destacando por un elevado potencial aromático con notas afrutadas y florales intensas [22].

El estudio de la composición aromática, así como de la influencia de las condiciones del entorno, en las variedades blancas poco conocidas, pueden ser de gran interés para la obtención de vinos con mayor complejidad aromática, que reflejen el carácter del terroir y presenten características de diferenciación en el mercado.

El objetivo de este trabajo fue el estudio de la influencia del entorno geográfico en el perfil aromático de la variedad Tempranillo Blanco en la D.O.Ca. Rioja (España).

\section{Material y métodos}

El estudio se llevó a cabo con la variedad Tempranillo Blanco durante la campaña 2016, en diferentes viñedos de la D.O.Ca. Rioja (España). Se seleccionaron siete parcelas experimentales distribuidas en las tres subzonas que integran la denominación, con características vitivinícolas diferenciadas debido a las condiciones de clima y suelo. En la subzona de Rioja Baja se localizaron dos parcelas (Alfaro y Corera), tres en Rioja Media (Logroño, Nalda y Albelda) y dos en Rioja Alta (Cenicero y Fonzaleche). Las condiciones climáticas recogidas en las estaciones meteorológicas más próximas (desde 1 de octubre de 2015 a 30 de septiembre de 2016) se muestran en la Figura 1.

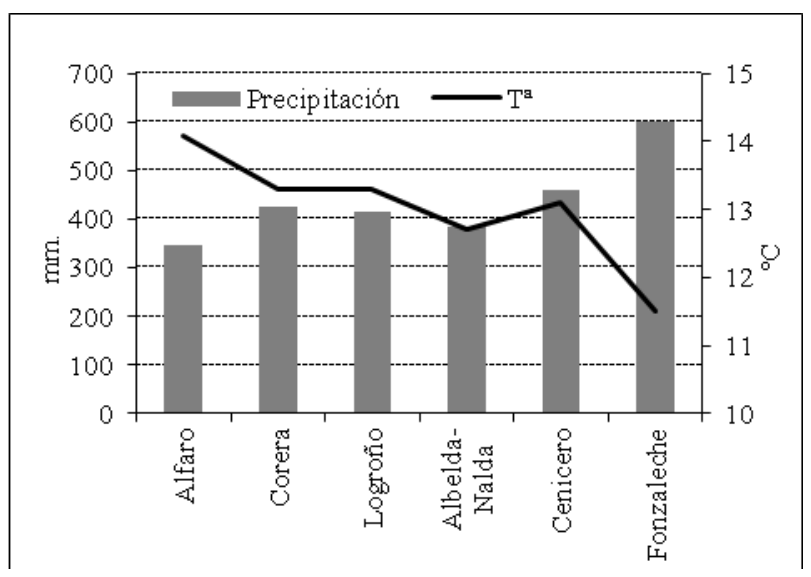

Figura 1. Condiciones climáticas en parcelas de Tempranillo Blanco.

Los viñedos fueron plantados entre los años 2008-2011, conducidos en espaldera, doble cordón Royat, con un marco de plantación de $2.90 \times 1.10 \mathrm{~m}$. Su altitud media osciló entre 345 y 555 m, con la excepción de una parcela en Rioja Media (Albelda) situada a $720 \mathrm{~m}$ de altitud.

La vendimia de la uva se efectuó al alcanzar la madurez tecnológica, entre los días 31 de agosto y 10 de octubre. En ese momento se evaluaron los parámetros siguientes: producción ( $\mathrm{kg}$ uva/cepa), peso medio del racimo (g) y de la baya (g). Previamente, se realizó el muestreo de 500 bayas, por triplicado, en cada una de las parcelas, que se separaron en dos lotes. 200 bayas se destinaron al análisis de la composición físico-química (grado probable, $\mathrm{pH}$, acidez total, ácidos tartárico y málico y potasio) según los métodos oficiales [23]. Las 300 bayas restantes se procesaron con una trituradora Thermomix ${ }^{\circledR}$ TM5 durante 15 segundos (velocidad 4) y el mosto obtenido se congeló a $-20^{\circ} \mathrm{C}$ para el posterior análisis de aromas varietales.

Los compuestos volátiles libres fueron determinados por microextracción en fase sólida y posterior cromatografía Gases-Masas (HS-SPME-GC-MS) Las muestras de uva fueron extraídas según el método descrito por [24] y modificado por [25]. La fibra SPME utilizada fue DVB/CAR/PDMS, 50/30 $\mu \mathrm{m}$. Las extracciones se realizaron en viales de vidrio de $20 \mathrm{ml}$, a los que se adicionó $9 \mathrm{ml}$ de muestra, $2.5 \mathrm{~g}$ de $\mathrm{NaCl}$ y $10 \mu \mathrm{l}$ de patrón interno (2-Octanol) en una concentración de $24.33 \mathrm{mg} / \mathrm{l}$. Después de agregar un imán de agitación el vial se colocó en el GC-MS. El acondicionamiento de la muestra se hizo a $60^{\circ} \mathrm{C}$, durante 15 minutos con agitación de $500 \mathrm{rpm}$. A continuación, la fibra SPME se insertó automáticamente en el espacio superior del vial de muestra, y se mantuvo durante $105 \mathrm{~min}$ con agitación. Una vez concluida la extracción, la fibra se introdujo en el puerto de inyección del GC a $250^{\circ} \mathrm{C}$ y se mantuvo durante 15 minutos para la 
desorción. Los compuestos desorbidos se separaron en un cromatógrafo de gases $7890 \mathrm{~B}$ con detector de masas de triple cuadrupolo (MS/MS) $7000 \mathrm{C}$ de Agilent Technologies (Palo Alto, CA, USA) conectado al programa Agilent MassHunter Qualitative Analysis B.07.00. Se usó helio como gas portador (1,2 $\mathrm{ml} / \mathrm{min})$. Las inyecciones se realizaron en modo splitless (1 min). Las condiciones cromatográficas fueron las descritas por [25]. El análisis de los compuestos C6 se llevó a cabo por el mismo procedimiento, pero con la muestra diluida $1 / 30$ con agua milliQ, ya que se encuentran en concentración muy elevada. Para la cuantificación de los compuestos se empleó el método semicuantitativo, en relación al área y la concentración del patrón interno empleado.

El estudio estadístico de los resultados se realizó mediante el análisis de varianza ANOVA y en caso de existir diferencias significativas, $\mathrm{p}<0.05$, se utilizó el test de Tukey para la separación de medias (programa estadístico SPSS versión 20.0). También se aplicó un análisis multivariante de componentes principales (programa InfoStat).

\section{Resultados y discusión}

Los parámetros productivos obtenidos para la variedad Tempranillo Blanco en las diferentes parcelas se muestran en la Tabla 1. Como puede observarse, se encontraron diferencias entre Logroño, donde se alcanzó la producción mayor, y Alfaro, Albelda y Nalda, con rendimientos más bajos. Los racimos de mayor tamaño se obtuvieron en Corera y Logroño, y los más pequeños en Alfaro y Nalda. En cuanto al tamaño de las bayas, las más grandes se encontraron en Nalda y las de menor tamaño en Alfaro. Este parámetro estuvo menos influenciado por las condiciones de cultivo, lo que parece indicar que se trata de una característica varietal.

Tabla 1. Parámetros productivos en parcelas de Tempranillo Blanco (2016). Media \pm desviación estándar $(n=3)$.

\begin{tabular}{|c|c|c|c|c|}
\hline 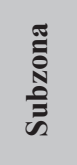 & 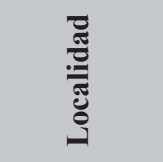 & 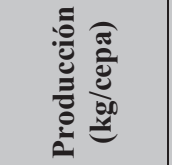 & 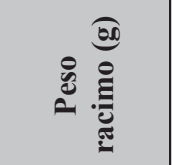 & 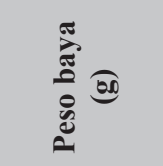 \\
\hline \multirow{2}{*}{ 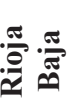 } & Alfaro & $3.10 \pm 0.28 \mathbf{b}$ & $150 \pm 0.90 \mathbf{b}$ & $1.37 \pm 0.08 \mathbf{c}$ \\
\hline & Corera & $4.61 \pm 0.87 \mathbf{a b}$ & $300 \pm 5.30 \mathbf{a}$ & $2.03 \pm 0.29 \mathbf{a b}$ \\
\hline \multirow{3}{*}{ 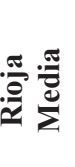 } & Logroño & $6.81 \pm 1.83 \mathbf{a}$ & $290 \pm 4.10 \mathbf{a}$ & $1.79 \pm 0.20 \mathbf{b c}$ \\
\hline & Albelda & $3.67 \pm 0.58 \mathbf{b}$ & $240 \pm 4.90 \mathbf{a b}$ & $1.85 \pm 0.72 \mathbf{b c}$ \\
\hline & Nalda & $3.53 \pm 0.15 \mathbf{b}$ & $180 \pm 1.70 \mathbf{b}$ & $2.38 \pm 0.17 \mathbf{a}$ \\
\hline \multirow{2}{*}{ 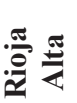 } & Cenicero & $4.32 \pm 1.15 \mathbf{a b}$ & $190 \pm 5.10 \mathbf{a b}$ & $1.63 \pm 0.25 \mathbf{b c}$ \\
\hline & Fonzaleche & $4.97 \pm 1.13 \mathbf{a b}$ & $220 \pm 2.20 \mathbf{a b}$ & $2.13 \pm 0.09 \mathbf{a b}$ \\
\hline
\end{tabular}

Letras diferentes en la misma columna indican diferencias según el test de Tukey $\mathrm{p}<0.05$.

La composición físico-química de la uva de Tempranillo blanco de las diferentes parcelas (Tabla 2) estuvo claramente influenciada por las condiciones climáticas, con la excepción del grado probable. No obstante, este parámetro mostró valores medios más bajos en la parcela de Albelda, en la que no alcanzó un nivel correcto de madurez, probablemente a causa de su elevada altitud. El $\mathrm{pH}$ más alto se obtuvo en la uva procedente de Corera debido a su elevado contenido de potasio, que podría estar relacionado con el tipo de suelo del viñedo. La acidez total fue superior en las parcelas de Rioja Alta (Cenicero y Fonzaleche) y más baja en Alfaro, debido a las condiciones climáticas en que se desarrolló la maduración.

Tabla 2. Composición físico-química de la uva de Tempranillo Blanco (2016). Media \pm desviación estándar ( $\mathrm{n}=3$ ).

\begin{tabular}{|c|c|c|c|c|c|c|c|}
\hline Subzona & Localidad & $\begin{array}{c}\text { Grado Prob. } \\
(\% \mathbf{v} / \mathbf{v})\end{array}$ & $\mathbf{p H}$ & $\begin{array}{c}\text { Acidez Total } \\
(\mathbf{g} / \mathbf{l})\end{array}$ & $\begin{array}{c}\text { Ac. Tartárico } \\
(\mathbf{g} / \mathbf{l})\end{array}$ & $\begin{array}{c}\text { Ac. Málico } \\
(\mathbf{g} / \mathbf{l})\end{array}$ & $\begin{array}{c}\text { Potasio } \\
(\mathbf{m g} / \mathbf{l})\end{array}$ \\
\hline \multirow{3}{*}{ Rioja Baja } & Alfaro & $12.9 \pm 0.10 \mathbf{a}$ & $3.48 \pm 0.04 \mathbf{a b}$ & $4.99 \pm 0.06 \mathbf{c}$ & $6.77 \pm 0.08 \mathbf{b c}$ & $2.17 \pm 0.06 \mathbf{b}$ & $1318 \pm 95 \mathbf{b c}$ \\
\cline { 2 - 8 } & Corera & $13.1 \pm 0.25 \mathbf{a}$ & $3.58 \pm 0.05 \mathbf{a}$ & $6.28 \pm 0.48 \mathbf{a b}$ & $8.32 \pm 0.08 \mathbf{a b}$ & $3.39 \pm 0.33 \mathbf{a}$ & $1786 \pm 83 \mathbf{a}$ \\
\hline \multirow{3}{*}{ Rioja Media } & Logroño & $13.0 \pm 0.41 \mathbf{a}$ & $3.43 \pm 0.06 \mathbf{a b}$ & $5.23 \pm 0.36 \mathbf{b c}$ & $7.32 \pm 0.34 \mathbf{b c}$ & $2.19 \pm 0.33 \mathbf{a b}$ & $1255 \pm 44 \mathbf{b c}$ \\
\cline { 2 - 8 } & Albelda & $11.5 \pm 0.04 \mathbf{a}$ & $3.23 \pm 0.04 \mathbf{b}$ & $5.78 \pm 0.33 \mathbf{b c}$ & $6.87 \pm 0.30 \mathbf{b c}$ & $1.91 \pm 0.17 \mathbf{b}$ & $832 \pm 46 \mathbf{d}$ \\
\cline { 2 - 8 } & Nalda & $13.9 \pm 0.35 \mathbf{a}$ & $3.30 \pm 0.06 \mathbf{b}$ & $5.48 \pm 0.11 \mathbf{b c}$ & $7.07 \pm 0.39 \mathbf{b c}$ & $2.19 \pm 0.18 \mathbf{b}$ & $1184 \pm 44 \mathbf{c}$ \\
\hline \multirow{2}{*}{ Rioja Alta } & Cenicero & $13.4 \pm 0.90 \mathbf{a}$ & $3.37 \pm 0.04 \mathbf{b}$ & $6.64 \pm 0.14 \mathbf{a}$ & $8.63 \pm 0.33 \mathbf{a}$ & $2.69 \pm 0.24 \mathbf{a b}$ & $1491 \pm 177 \mathbf{b}$ \\
\cline { 2 - 8 } & Fonzaleche & $13.7 \pm 0.14 \mathbf{a}$ & $3.17 \pm 0.02 \mathbf{b}$ & $6.89 \pm 0.02 \mathbf{a}$ & $6.76 \pm 0.24 \mathbf{c}$ & $2.93 \pm 0.04 \mathbf{a b}$ & $1193 \pm 12 \mathbf{c}$ \\
\hline
\end{tabular}

Letras diferentes en la misma columna indican diferencias según el test de Tukey $\mathrm{p}<0.05$

En el análisis de la composición volátil varietal de Tempranillo Blanco se detectaron 43 compuestos, que pueden agruparse en 8 familias químicas (alcoholes, aldehídos y cetonas, terpenos, norisoprenoides, ésteres, ácidos, compuestos C6 y bencenoides). En la Tabla 3 se muestra la abundancia relativa de cada uno de los grupos de compuestos identificados en las diferentes parcelas. En todos los casos, los norisoprenoides fueron los compuestos más abundantes (48-70\%), seguidos por los bencenoides $(8-29 \%)$ y los aldehídos y cetonas (3-18\%). En menor proporción se encontraron los compuestos C6 $(5-16 \%)$, alcoholes $(2-15 \%)$, terpenos $(2-5 \%)$ y otros (ácidos, ésteres...). En Alfaro los norisoprenoides fueron mucho más abundantes que en el resto de parcelas, 
probablemente porque la temperatura fue más alta, tal como ha sido observado por otros autores [13]. En Nalda y Albelda (parcelas muy próximas, pero con diferente altitud) se encontró el mayor porcentaje de bencenoides; y en Corera y Fonzaleche destacaron los aldehídos y cetonas y los alcoholes. En el viñedo de Logroño se obtuvieron más compuestos C6, lo que podría deberse a la elevada producción obtenida. Las diferencias en el porcentaje de terpenos relacionadas con la ubicación del viñedo fueron muy escasas, lo que indicaría que se trata de un carácter varietal.

Tabla 3. Composición volátil (\%) en parcelas de Tempranillo Blanco (2016).

\begin{tabular}{|c|c|c|c|c|c|c|c|}
\hline \multirow[b]{2}{*}{ Familia química } & \multicolumn{2}{|c|}{ Rioja Baja } & \multicolumn{3}{|c|}{ Rioja Media } & \multicolumn{2}{|c|}{ Rioja Alta } \\
\hline & 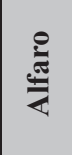 & نัँّ & 苞 & $\begin{array}{l}\frac{\pi}{0} \\
\frac{0}{0} \\
\frac{0}{2}\end{array}$ & $\frac{\frac{\pi}{0}}{\frac{\pi}{\pi}}$ & 这 & 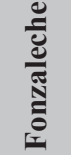 \\
\hline Norisoprenoides & 70.3 & 49.1 & 51.1 & 53.3 & 50.7 & 50.4 & 47.6 \\
\hline Bencenoides & 8.53 & 8.29 & 13.7 & 28.5 & 20.2 & 16.7 & 13.3 \\
\hline \begin{tabular}{|l} 
Aldehídos y \\
Cetonas
\end{tabular} & 5.84 & 17.4 & 10.1 & 3.27 & 11.5 & 12.8 & 17.9 \\
\hline Compuestos C6 & 8.41 & 4.94 & 16.0 & 9.90 & 5.34 & 6.56 & 5.69 \\
\hline Alcoholes & 2.89 & 15.1 & 3.94 & 1.90 & 8.41 & 8.43 & 11.2 \\
\hline Terpenos & 3.23 & 4.27 & 4.75 & 2.26 & 3.29 & 3.30 & 3.78 \\
\hline Ácidos grasos & 0.32 & 0.63 & 0.23 & 0.56 & 0.30 & 1.11 & 0.19 \\
\hline Ésteres & 0.50 & 0.38 & 0.21 & 0.30 & 0.30 & 0.70 & 0.24 \\
\hline
\end{tabular}

El contenido total de aromas varietales (Figura 2), así como el de la mayor parte de las familias químicas analizadas (Tabla 3), fue superior en la uva procedente de la parcela de Nalda, mientras que los valores más bajos se encontraron en Logroño, Alfaro y Albelda. Los resultados obtenidos no mostraron una clara relación entre la localización geográfica del viñedo según la clasificación por subzonas de la D.O.Ca. Rioja y el contenido de aromas varietales de la uva. En Rioja Baja el contenido aromático fue mucho más bajo en Alfaro que en Corera, probablemente debido a las diferencias climáticas entre ambas parcelas. En Rioja Media las diferencias entre las parcelas se acentuaron más, a pesar de su proximidad geográfica y climatología similar, y podrían estar relacionadas con la producción y la altitud, además de la interacción con otros muchos factores no considerados en este estudio (tipo de suelo, exposición...) [13]. En Rioja Alta el contenido aromático alcanzó valores medios, algo más bajos en Fonzaleche, donde las precipitaciones fueron las más elevadas y las temperaturas más bajas.

Los norisoprenoides y los terpenos son los compuestos más odoríferos, presentan aromas florales y cítricos que juegan un papel clave en el aroma varietal de los vinos [2]. En Tempranillo Blanco se detectaron 8 norisoprenoides, entre los que destacaron por su mayor concentración los dos isómeros de $\beta$-damascenona, $\beta$ ionona y dehidro- $\beta$-ionona (Tabla 4). Los valores más elevados correspondieron al viñedo de Nalda, aunque con pocas diferencias respecto a Corera y Cenicero.

El contenido de compuestos terpénicos fue en general bajo, se cuantificaron 6 compuestos pertenecientes a este grupo, entre los que destacaron acetona de geranilo, pcymenene, $\mathrm{p}$-cymene y linalol. Todos ellos, junto con $\alpha$ terpineol y limoneno tuvieron mayor concentración en la uva procedente de las parcelas de Nalda y Corera.

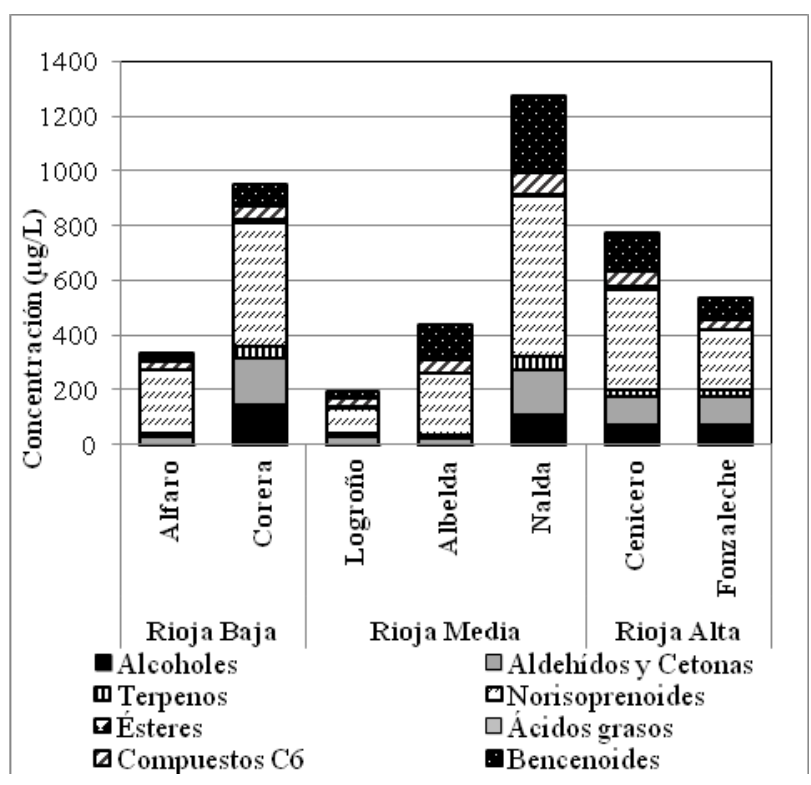

Figura 2. Composición volátil total $(\mu \mathrm{g} / \mathrm{l})$ de Tempranillo Blanco en diferentes parcelas (año 2016).

En el grupo de los bencenoides se detectaron 3 compuestos, 2-feniletanal, benzaldehído y 2-feniletanol. Estos últimos puede contribuir positivamente al aroma del vino aportando notas dulces y florales (rosa), y se forman principalmente durante la fermentación alcohólica [7]. Estos compuestos fueron significativamente más elevados en el viñedo de Nalda, y apenas se observaron diferencias entre el resto de parcelas.

Entre los volátiles clasificados como C6 se detectaron 6 compuestos, dos de los cuales (hexanal y E,E-2,4hexadienal) tuvieron concentraciones mayores al resto. Este grupo de sustancias son responsables de aromas verdes y herbáceos, y dependiendo de su concentración pueden afectar negativamente a la calidad del vino [7]. $\mathrm{Su}$ formación se produce principalmente durante las etapas prefermentativas de la vinificación. Los resultados observados por [19] en diferentes variedades blancas mostraron que estos compuestos fueron los más abundantes (70-93\%) en los mostos obtenidos después del estrujado y prensado de la uva.

En el análisis de componentes principales (Figura 3) las dos primeras funciones explicaron el $98 \%$ de la varianza, y permitieron la clasificación de las parcelas de Tempranillo Blanco en función de su composición 
volátil. La función 1 explicó el $83.7 \%$ de la varianza, y se correlacionó positivamente con los terpenos y norisoprenoides. En la parte positiva del eje X se posicionaron las parcelas de Nalda y Corera, en la negativa Alfaro, Logroño y Albelda. 
Tabla 4. Composición volátil $(\mu \mathrm{g} / \mathrm{l})$ en parcelas de Tempranillo Blanco (2016). Media \pm desviación estándar $(\mathrm{n}=3)$

\begin{tabular}{|c|c|c|c|c|c|c|c|}
\hline \multirow{2}{*}{ Compuesto } & \multicolumn{2}{|c|}{ Rioja Baja } & \multicolumn{3}{|c|}{ Rioja Media } & \multicolumn{2}{|c|}{ Rioja Alta } \\
\hline & Alfaro & Corera & Logroño & Nalda & Albelda & Cenicero & Fonzaleche \\
\hline$\beta$-Ciclocitral & $2,89 \pm 0,95 \mathbf{a}$ & $5,86 \pm 1,02 \mathbf{a}$ & $3,55 \pm 1,41 \mathbf{a}$ & $5,68 \pm 1,36 \mathbf{a}$ & $2,57 \pm 0,54 \mathbf{a}$ & $5,25 \pm 1,95 \mathbf{a}$ & $5,21 \pm 2,16 \mathbf{a}$ \\
\hline$(Z)-\beta$ - Damascenona & $12,03 \pm 1,56 \mathbf{b c}$ & $23,83 \pm 2,24 \mathbf{a b}$ & $4,91 \pm 0,74 \mathbf{c}$ & $30,93 \pm 12,40 \mathbf{a}$ & $14,95 \pm 5,68 \mathbf{a b c}$ & $21,01 \pm 4,49 \mathbf{a b c}$ & $13,99 \pm 5,35 \mathbf{b c}$ \\
\hline (E)- $\beta$ - Damascenona & $201 \pm 7,87 \mathbf{b c}$ & $387 \pm 23,00 \mathbf{a b}$ & $76,6 \pm 13,96 \mathbf{c}$ & $495 \pm 173 \mathbf{a}$ & $190 \pm 45 \mathbf{b c}$ & $300 \pm 26 \mathbf{a b c}$ & $169 \pm 116 \mathbf{b c}$ \\
\hline$\beta$-Ionona & $7,67 \pm 2,71 \mathbf{b}$ & $15,72 \pm 3,15 \mathbf{a b}$ & $8,85 \pm 3,37 \mathbf{a b}$ & $17,45 \pm 4,57 \mathbf{a}$ & $6,36 \pm 1,14 \mathbf{b}$ & $14,45 \pm 3,82 \mathbf{a b}$ & $10,46 \pm 4,15 \mathbf{a b}$ \\
\hline Dehidro- $\beta$-ionona & $3,91 \pm 0,33 \mathbf{b}$ & $16,29 \pm 3,08 \mathbf{a b}$ & $2,38 \pm 0,40 \mathbf{b}$ & $35,48 \pm 16,30 \mathbf{a}$ & $7,20 \pm 2,96 \mathbf{b}$ & $18,92 \pm 4,39 \mathbf{a b}$ & $19,99 \pm 13,64 \mathbf{a b}$ \\
\hline Vianol & $0,42 \pm 0,03 \mathbf{a}$ & $2,63 \pm 2,26 \mathbf{a}$ & $0,14 \pm 0,07 \mathbf{a}$ & $1,69 \pm 1,32 \mathbf{a}$ & $0,56 \pm 0,41 \mathbf{a}$ & $0,99 \pm 0,44 \mathbf{a}$ & $0,63 \pm 0,15 \mathbf{a}$ \\
\hline$\beta$-Maaliene & $0,26 \pm 0,09 \mathbf{a}$ & $0,35 \pm 0,15 \mathbf{a}$ & $0,12 \pm 0,04 \mathbf{a}$ & $0,20 \pm 0,03 \mathbf{a}$ & $0,18 \pm 0,15 \mathbf{a}$ & $0,44 \pm 0,20 \mathbf{a}$ & $0,17 \pm 0,09 \mathbf{a}$ \\
\hline $\begin{array}{l}\text { Metil- } \\
\text { dihidroepijasmonato }\end{array}$ & $1,39 \pm 0,33 \mathbf{a}$ & $1,46 \pm 0,25 \mathbf{a}$ & $0,10 \pm 0,02 \mathbf{b}$ & $1,44 \pm 0,73 \mathbf{a}$ & $0,16 \pm 0,04 \mathbf{b}$ & $1,03 \pm 0,26 \mathbf{a b}$ & $0,63 \pm 0,44 \mathbf{a b}$ \\
\hline$\Sigma$ Norisoprenoides & $229 \pm 70 b c$ & $453 \pm 134 a b$ & $97 \pm 26,2 c$ & $588 \pm 171 \mathrm{a}$ & $222 \pm 66 b c$ & $363 \pm 103 b$ & $220 \pm 57 b c$ \\
\hline Benzaldehído & $5,96 \pm 6,17 \mathbf{c}$ & $29,52 \pm 5,15 \mathbf{b c}$ & $5,73 \pm 2,36 \mathbf{c}$ & $107,89 \pm 18,60 \mathbf{a}$ & $29,90 \pm 7,69 \mathbf{b c}$ & $52,35 \pm 13,45 \mathbf{b}$ & $30,76 \pm 10,93 \mathbf{b c}$ \\
\hline 2-Feniletanal & $21,59 \pm 7,86 \mathbf{d}$ & $48,72 \pm 0,91 \mathbf{c d}$ & $20,21 \pm 10,57 \mathbf{d}$ & $163,94 \pm 28,75 \mathbf{a}$ & $99,03 \pm 23,21 \mathbf{b}$ & $82,51 \pm 20,49 \mathbf{b c}$ & $45,08 \pm 8,75 \mathbf{c d}$ \\
\hline 2-Feniletanol & $1,43 \pm 0,34 \mathbf{b}$ & $3,72 \pm 1,43 \mathbf{b}$ & $1,02 \pm 0,37 \mathbf{b}$ & $11,96 \pm 1,84 \mathbf{a}$ & $3,46 \pm 0,46 \mathbf{b}$ & $2,15 \pm 0,68 \mathbf{b}$ & $3,91 \pm 1,28 \mathbf{b}$ \\
\hline$\Sigma$ Bencenoides & $28,98 \pm 10,6 c$ & $81,96 \pm 22,6 b c$ & $26,97 \pm 10,0 c$ & $283,79 \pm 77 a$ & $132,39 \pm 49 b$ & $137,01 \pm 41 b$ & $79,75 \pm 21 b c$ \\
\hline Limoneno & $0,57 \pm 0,17 \mathbf{b}$ & $1,96 \pm 0,72 \mathbf{a}$ & $0,61 \pm 0,19 \mathbf{b}$ & $2,02 \pm 0,48 \mathbf{a}$ & $1,03 \pm 0,26 \mathbf{a b}$ & $1,15 \pm 0,39 \mathbf{a b}$ & $0,96 \pm 0,44 \mathbf{a b}$ \\
\hline p-Cymene & $1,19 \pm 0,69 \mathbf{b}$ & $5,28 \pm 0,76 \mathbf{a}$ & $0,82 \pm 0,22 \mathbf{b}$ & $4,90 \pm 1,28 \mathbf{a}$ & $1,16 \pm 0,37 \mathbf{b}$ & $3,57 \pm 1,84 \mathbf{a b}$ & $3,00 \pm 2,44 \mathbf{a b}$ \\
\hline p-Cymenene & $0,86 \pm 0,06 \mathbf{b c}$ & $4,73 \pm 1,11 \mathbf{a b}$ & $0,58 \pm 0,08 \mathbf{c}$ & $7,23 \pm 2,08 \mathbf{a}$ & $1,27 \pm 0,10 \mathbf{b c}$ & $2,99 \pm 0,76 \mathbf{b c}$ & $4,36 \pm 2,77 \mathbf{a b c}$ \\
\hline Linalol & $2,27 \pm 0,41 \mathbf{c d}$ & $3,98 \pm 0,59 \mathbf{a b}$ & $1,45 \pm 0,26 \mathbf{d}$ & $4,55 \pm 0,51 \mathbf{a}$ & $1,66 \pm 0,30 \mathbf{d}$ & $3,19 \pm 0,48 \mathbf{b c}$ & $2,17 \pm 0,52 \mathbf{c d}$ \\
\hline$\alpha$-Terpineol & $1,51 \pm 0,08 \mathbf{b}$ & $3,01 \pm 0,68 \mathbf{a b}$ & $0,63 \pm 0,22 \mathbf{b}$ & $6,25 \pm 3,00 \mathbf{a}$ & $1,20 \pm 0,21 \mathbf{b}$ & $3,20 \pm 0,56 \mathbf{a b}$ & $3,05 \pm 1,81 \mathbf{a b}$ \\
\hline Acetona de geranilo & $4,56 \pm 2,57 \mathbf{b}$ & $23,24 \pm 8,5 \mathbf{a}$ & $5,28 \pm 0,80 \mathbf{b}$ & $21,35 \pm 9,87 \mathbf{a}$ & $4,19 \pm 0,61 \mathbf{b}$ & $11,94 \pm 2,21 \mathbf{a b}$ & $9,14 \pm 5,02 \mathbf{a b}$ \\
\hline$\Sigma$ Terpenos & $10,96 \pm 1,46 b$ & $42,20 \pm 8,0 \mathrm{a}$ & $9,36 \pm 1,85 b$ & $46,30 \pm 6,91 \mathrm{a}$ & $10,52 \pm 1,21 b$ & $26,05 \pm 3,82 \mathrm{ab}$ & $22,69 \pm 2,85 a b$ \\
\hline Hexanal & $8,04 \pm 1,14 \mathbf{a}$ & $9,46 \pm 3,56 \mathbf{a}$ & $10,76 \pm 1,90 \mathbf{a}$ & $13,63 \pm 1,51 \mathbf{a}$ & $19,16 \pm 16,13 \mathbf{a}$ & $12,54 \pm 6,39 \mathbf{a}$ & $6,16 \pm 1,52 \mathbf{a}$ \\
\hline (Z)-3-Hexen-1-ol & $0,87 \pm 0,13 \mathbf{a}$ & $1,20 \pm 0,04 \mathbf{a}$ & $0,82 \pm 0,10 \mathbf{a}$ & $1,51 \pm 0,63 \mathbf{a}$ & $1,49 \pm 1,25 \mathbf{a}$ & $1,66 \pm 0,59 \mathbf{a}$ & $0,56 \pm 0,14 \mathbf{a}$ \\
\hline 1-Hexanol & $2,98 \pm 0,50 \mathbf{a}$ & $5,63 \pm 0,99 \mathbf{a}$ & $2,71 \pm 0,34 \mathbf{a}$ & $9,89 \pm 1,63 \mathbf{a}$ & $9,07 \pm 8,83 \mathbf{a}$ & $2,80 \pm 0,44 \mathbf{a}$ & $2,00 \pm 0,39 \mathbf{a}$ \\
\hline (E)-2-Hexen-1-ol & $1,56 \pm 0,37 \mathbf{c d}$ & $3,30 \pm 0,30 \mathbf{b c}$ & $1,53 \pm 0,63 \mathbf{c d}$ & $5,20 \pm 0,91 \mathbf{a}$ & $1,26 \pm 1,16 \mathbf{d}$ & $4,15 \pm 0,43 \mathbf{a b}$ & $1,50 \pm 0,19 \mathbf{c d}$ \\
\hline (E)-2-Hexenal & $4,94 \pm 0,95 \mathbf{b}$ & $8,33 \pm 0,78 \mathbf{a b}$ & $7,81 \pm 2,07 \mathbf{a b}$ & $8,98 \pm 5,62 \mathbf{a b}$ & $4,11 \pm 3,58 \mathbf{b}$ & $13,23 \pm 1,2 \mathbf{a}$ & $4,62 \pm 1,21 \mathbf{b}$ \\
\hline E,E-2,4-Hexadienal & $10,16 \pm 0,63 \mathbf{b}$ & $20,97 \pm 3,18 \mathbf{b}$ & $7,82 \pm 2,68 \mathbf{b}$ & $35,96 \pm 8,19 \mathbf{a}$ & $10,88 \pm 1,26 \mathbf{b}$ & $19,66 \pm 3,7 \mathbf{b}$ & $19,31 \pm 7,75 \mathbf{b}$ \\
\hline$\Sigma$ Compuestos C6 & $28,55 \pm 3,70 b$ & $48,89 \pm 6,99 \mathrm{ab}$ & $31,45 \pm 4,09 b$ & $75,17 \pm 12,20 a$ & $45,97 \pm 6,87 b$ & $54,04 \pm 7,21 \mathrm{ab}$ & $34,16 \pm 6,99 b$ \\
\hline
\end{tabular}

Letras diferentes en la misma fila indican diferencias según el test de Tukey $p \quad<0.05$

La función 2 explicó un $14.31 \%$ de la varianza y se correlacionó positivamente con el contenido en bencenoides. Según esta función en la parte positiva del eje Y se posicionaron las parcelas de Albelda y Nalda, y en la negativa Corera. Las parcelas de Cenicero y Fonzaleche se situaron en la zona intermedia par ambas funciones. 


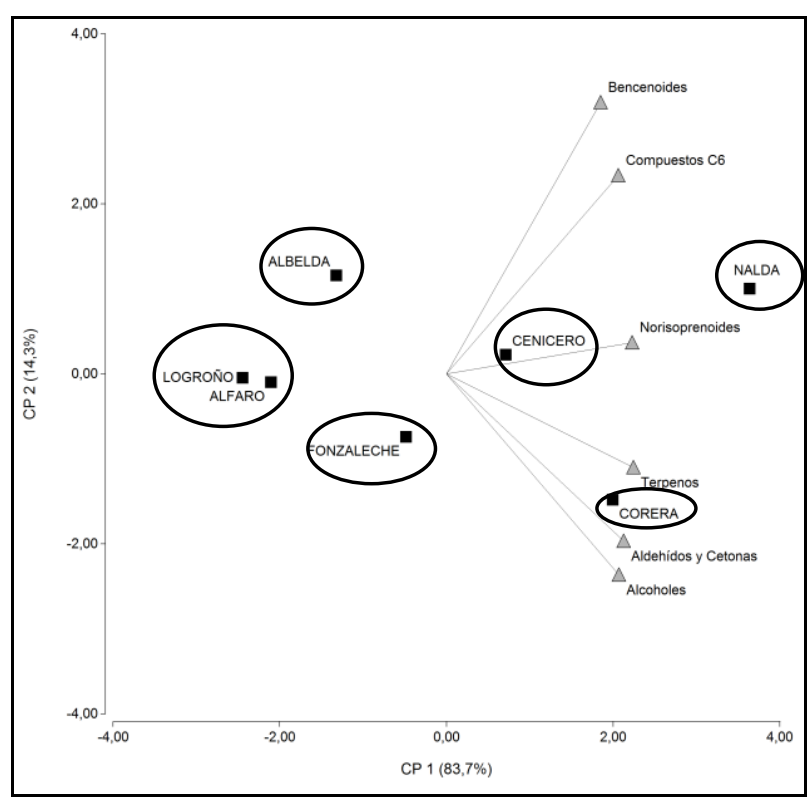

Figura 3. Componentes principales de la composición volátil de Tempranillo Blanco en diferentes parcelas (año 2016).

\section{Conclusiones}

En este trabajo se cuantificó por primera vez la composición volátil de la variedad Tempranillo Blanco en siete localizaciones geográficas de la D.O.Ca. Rioja, durante la campaña 2016. Los resultados obtenidos mostraron diferencias notables en el contenido aromático de la uva procedente de las distintas ubicaciones del viñedo, pero no se pudo establecer ninguna relación con la clasificación por subzonas de la D.O.Ca. Rioja. A pesar de las diferencias observadas, el perfil aromático varietal se mantuvo, lo que parece indicar que Tempranillo Blanco es una variedad con amplias posibilidades de adaptación a diferentes entornos vitícolas.

Finalmente, es preciso remarcar la necesidad de continuar estos estudios en otras campañas, con condiciones climáticas muy diferentes, con la finalidad de ampliar los conocimientos acerca de la influencia del entorno sobre el perfil volátil de Tempranillo Blanco, lo que permitirá confirmar sus posibilidades para la elaboración de vinos blancos con características diferenciadas que reflejen el carácter del terroir.

\section{Bibliografía}

1. Z. Gunata, C. Bayonove, R. Baumes, R Cordonnier, J. Chromatogr. 331, 83-90 (1985)

2. P. Ribéreau-Gayon, Y. Glories, A. Maujean, D. Dubourdieu, Handbook of Enology. The Chemistry of Wine, Vol 2 (Chichester: John Wiley and Sons Ltd, 2006)

3. M.J. Cabrita, A.M. Freitas, O. Laureano, R. di Stefano, J. Cromatogr. A 778, 219-224 (1997)

4. S.M. Bureau, A.L. Rzungles, R.L. Baumes, J. Sci. Food Agric. 80, 2012-2020 (2000)

5. M.A. Sefton, I.L. Francis, P.J. Williams, Am. J. Enol. Vitic. 44, 359-370 (1993)
6. M.A. Sefton, I.L. Francis, P.J. Williams, J. Food Sci. 59, 142-147 (1994)

7. A. Pedroza, A. Zalacain, J.F. Lar, M.R. Salinas, Food Res Int. 43, 1003-1008 (2010)

8. J.M. Oliveira, I. Araujo, O. Pereira, S. Maia, A.J. Amaral, M.O. Maia, Anal. Chim. Acta 513, 269275 (2004)

9. J.M. Oliveira, M. Faria, F. Sa, F. Barroa, I. Araujo, Anal. Chim. Acta 563, 300-309A (2006)

10. A. Panighel, R. Flamini, Molecules 40 (19), 21291 21309 (2014)

11. E. Sánchez-Palomo, R. Alonso-Villegas, M.A. González Viñas, Food Chem. 173, 1195-1202 (2015)

12. A. Zalacain, J. Marín, G.L. Alonso, M.R. Salinas, Talanta 71(4), 1610-1615 (2007)

13. J. Marais, G. Versini, C. Wyk, A. Rapp, S. Afr. J. Enol. Vitic. 13, 71-77 (1992)

14. A.L. Robinson, P.K. Boss, P.S. Solomon, R.D. Trengove, H. Heymann, S.E. Ebeler, Am. J. Enol. Vitic. 65, 1-23 (2014)

15. C. Peyrot des Gachons, C. van Leeuwen, T. Tominaga, G.P. Soyer, J.P. Gaudillère, D. Dubourdieu, J. Sci. Food Agric. 85, 73-85 (2005)

16. T. Tominaga, C. Peyrot des Gachons, D. Dubourdieu, J. Agric. Food Chem. 46, 5215-5219 (1998)

17. M. Vilanova, Z. Genisheva, M. Graña, J.M. Oliveira, S. Afr. J. Enol. Vitic. 34 (2), 212-222 (2013)

18. J.E. Bueno, R. Peinado, J. Moreno, M. Medina, L. Moyano, L. Zea, J. Food Sci. 68(1), 158-163 (2003)

19. A. Gonzalo-Diago, E. Terroba -Pérez, E. Baroja, E. García-Escudero, J. Martínez, Abstracts Macrowine, 200 (2016)

20. E. López-Tamames, N. Carro-Mariño, Y. Gunata, C. Sapis, R. Baumes, C. Bayonove. J. Agric. Food Chem. 45(5), 1729-1735 (1997)

21. J. Martínez, T. Vicente, T. Martínez, J.B. Chávarri, E. García-Escudero. Actas XXIX Congreso Mundial de la Viña y el Vino,1, 41-43 (2006)

22. J. Martínez, A. Gonzalo, E. Baroja, E. GarcíaEscudero, Enoviticultura 47, 2-10 (2017)

23. ECC, Official Journal of the European Comunity, L272 (3), 1-192 (1990)

24. R. Perestrelo, A.S. Barros, S.M. Rocha, J.S. Câmara. Talanta 85,1483-1493 (2011)

25. T. Garde-Cerdán, L. González-Arenzana, N. López, R. López, P. Santamaría, I. López-Alfaro. Innov. Food Sci. Emerg. Technol. 20, 91-99 (2013)

\section{Agradecimientos}

Este trabajo ha sido realizado mediante un proyecto de investigación cofinanciado al $50 \%$ por el Gobierno de La Rioja y Fondos FEDER durante 2016 (Programa Operativo FEDER de La Rioja 2014-2020) 